\title{
Tailored Retrieval of Health Information from the Web for Facilitating Communication and Empowerment of Elderly People
}

\author{
Marco Alfano ${ }^{1,5}$, Biagio Lenzitti ${ }^{2}$, Davide Taibi ${ }^{3}$ and Markus Helfert ${ }^{4}$ \\ ${ }^{1}$ Lero, Dublin City University, Dublin, Ireland \\ ${ }^{2}$ Dipartimento di Matematica e Informatica, Università di Palermo, Palermo, Italy \\ ${ }^{3}$ Istituto per le Tecnologie Didattiche, Consiglio Nazionale delle Ricerche, Palermo, Italy \\ ${ }^{4}$ Lero, Maynooth University, Maynooth, Co. Kildare, Ireland \\ ${ }^{5}$ Anghelos Centro Studi sulla Comunicazione, Palermo, Italy \\ marco.alfano@lero.ie,biagio.lenzitti@unipa.it,davide.taibi@itd.cnr.it,markus.helfert@lero.ie
}

Keywords: e-Health, Patient Empowerment, Communication, Health Information Seeking, User Requirements, Structured Data, Search Engine.

\begin{abstract}
A patient, nowadays, acquires health information from the Web mainly through a "human-to-machine" communication process with a generic search engine. This, in turn, affects, positively or negatively, his/her empowerment level and the "human-to-human" communication process that occurs between a patient and a healthcare professional such as a doctor. A generic communication process can be modelled by considering its syntactic-technical, semantic-meaning, and pragmatic-effectiveness levels and an efficacious communication occurs when all the communication levels are fully addressed. In the case of retrieval of health information from the Web, although a generic search engine is able to work at the syntactic-technical level, the semantic and pragmatic aspects are left to the user and this can be challenging, especially for elderly people. This work presents a custom search engine, FACILE, that works at the three communication levels and allows to overcome the challenges confronted during the search process. A patient can specify his/her information requirements in a simple way and FACILE will retrieve the "right" amount of Web content in a language that he/she can easily understand. This facilitates the comprehension of the found information and positively affects the empowerment process and communication with healthcare professionals.
\end{abstract}

\section{INTRODUCTION}

According to the World Health Organization (WHO), empowerment is "a process through which people gain greater control over decisions and actions affecting their health" (WHO, 1998). It includes, as a basic step, the acquisition of health/medical information that helps patients/citizens to understand medical conditions and treatments, acquire selfconfidence to discuss them with medical professionals and, together, make the best-informed decisions (Akerkar \& Bichile, 2004; Smith, 2004).

The main source of health/medical information is, nowadays, the World Wide Web (or Web, for short) with the number of Web health information seekers that have been steadily increasing over the years (Pew Research Center, 2013; Taylor, 2010). Search engines are more and more used as the main tools to provide Web information. However, generic search engines do not make any distinction among the users and overload them with the amount of information. The use of a search engine for Web information retrieval entails a human-to-machine communication process between the user (e.g., a patient) and the search engine. It affects, among others, the amount, comprehension and use of the found information. This, in turn, may facilitate or complicate the humanto-human communication process between patients and healthcare professionals such as doctors (Smith, 2004).

Communication processes have been modelled in various ways in the past decades. One of the most famous communication model is the one introduced by Morris in relation to its theory of signs (Morris, 1938). It is made up of three levels, i.e., syntactic, semantic and pragmatic. It is paired up by another communication model introduced by Shannon and Weaver (Shannon, 1949) that tackles the problems of communication at the technical, semantic and effectiveness levels. The connection of the two 
models, as shown in the next section, leads to the following three levels (top down):

- Pragmatic - Effectiveness;

- Semantic - Meaning;

- Syntactic - Technical.

Although a complete communication process should take place in order to allow patients/citizens to fully understand and efficaciously use the found information, a quick analysis of the three-level communication model shows how a generic search engine is only able to retrieve information from the Web working at the syntactic-technical level, leaving the semantic and pragmatic part of the communication process in charge of the user. Elderly people, in particular, may have great difficulties in expressing their requirements, and understanding and using the received information, as envisaged by the semantic and pragmatic communication levels. This prevents them, for example, from having a true understanding of their medical conditions (semanticmeaning level) and acquire the self-confidence to communicate with their doctors and make a shared and informed decision (pragmatic-effectiveness level). Since a generic search engine is not able to work at the three communication levels, it does not constitute a real aid in the process of empowering a patient/citizen, especially an elderly one. It can also complicate the communication between a patient and a healthcare professional. For example, the different ways of dealing with the information found on the Web can lead to an argument between a patient and a doctor.

This work presents the characteristics and use of a custom search engine, FACILE, that has been created in order to satisfy the user information needs and overcome the communication challenges confronted during the search process. In particular, FACILE allows a user to specify his/her information requirements in a simple way. It, then, retrieves tailored Web information by exploiting the Web semantic capabilities provided by healthlifesci.schema.org structured data. By doing so, FACILE provides the "right" amount of Web content, without overwhelming the user, and in a language that he/she can easily understand. This positively affects patient's comprehension of conditions and treatment alternatives and, ultimately, facilitates, his/her empowerment process.

The paper is organized as follows. Section 2 illustrates the problems and challenges presented by the retrieval of Web health information in relation to the expected communication process, together with

\footnotetext{
${ }^{1}$ http://www.ncbi.nlm.nih.gov/pubmed/
}

the motivation of the present work. Section 3 presents the characteristics and implementation details of the FACILE custom engine that overcomes the problems and challenges presented in Section 2. Section 4 presents the use and experimental results of FACILE. Sections 5 and 6 present a discussion of the obtained results and some conclusions.

\section{BACKGROUND AND MOTIVATION}

"Engaging and empowering people \& communities" constitutes the first of the five strategies of the "Framework on integrated people-centred health services" of WHO (WHO, 2016). It calls for a paradigm shift on the relation between patients/citizens and health. In fact, empowered patients have the necessary knowledge, skills, attitudes and self-awareness about their condition to understand their lifestyle and treatment options, make informed choices about their health and have control over the management of their condition/health in their daily life (European Health Parliament, 2017; Alfano et al., 2019a; Alfano et al., 2019b; Bodolica et al., 2019; Bravo et al., 2015, Cerezo et al., 2016; Fumagalli et al., 2015).

As seen in Section 1, the acquisition of medical/health information is a basic step in the empowerment process and the main source of health/medical information is, nowadays, the Web (Pew Research Center, 2013; Taylor, 2010; UK national statistics, 2010; Instituto Nacional de Estadística, 2010). Search engines are the main tools used to retrieve information from the Web (Pletneva, 2011; Roberts, 2017). However, generic search engines do not make any distinction among the users and overload them with a huge amount of information that is often outdated or of poor quality. Moreover, the Web is full of information not easily understandable since users, such as patients/citizens, lack a specific expertise in the health domain. Although, some of these problems might be overcome with the advanced features of a search engine, generic users, and mainly elderly people, do not usually have the skills required to use such features and avoid these problems. Specialized search engines $\left(\right.$ PubMed $^{1}$ or Quertle ${ }^{2}$ ), on the other hand, mainly work on medical literature and result quite complex for generic users, and especially the elderly ones. Finally, specialized health/medical websites

\footnotetext{
${ }^{2}$ https://quertle.com/
} 
(e.g., WebMD ${ }^{3}$, MedlinePlus $^{4}$ or Health on Net Foundation Select ${ }^{5}$ ) are mainly built by hand so presenting a limited and often outdated amount of information (compared to what is available on the Web). Moreover, they are often not free.

The retrieval of health information from the Web requires a communication process between a user (an elderly patient/citizen in our case) and a search engine. Notice that a "complete" communication process, usually, entails different levels of communication. Many communication models exist in the literature and a very famous model (if not the most famous) is the one introduced by Morris in relation to his theory of signs (Morris, 1938). It is made up of three levels, i.e., syntactic, semantic and pragmatic and it has been used in several works dealing with human communication (Hahn \& Paynton, 2014; Cherry, 1966; Johnson \& Klare, 1961). Shannon and Weaver, on the other hand, present a mathematical theory of communication that is focused on information transmission (Shannon, 1949). Even though they mainly deal with the technical aspects of communication, they introduce other two levels above the technical one, i.e., the semantic and effectiveness levels, that are influenced by the technical level.

Interestingly enough, the two models have been connected by (Carlile, 2004) and an equivalence between the terms at the three levels has been established, in practice. We can then consider a "unified" communication model that presents the following levels (with the "semantic" term that has been associated to "meaning" as indicated in Watzlawick et al., 1967):

- Pragmatic-Effectiveness: How effectively does the received information affect behaviour?

- Semantic-Meaning: How precisely is the meaning conveyed?

- Syntactic-Technical: How accurately can the information be transmitted?

This communication model can be used, in principle, for both human-to-human communication (e.g., patient to doctor) and human-to-machine communication (e.g., patient to machine), as shown in Fig. 1. The human-to-human communication mainly deals with the syntactic-semantic-pragmatic aspects of the communication model, whereas the human-to-machine communication mainly deals with the technical-meaning-effectiveness aspects.

As seen in the Introduction, this work deals with tailored retrieval of health information for user

\footnotetext{
${ }^{3} \mathrm{http}: / / \mathrm{www} . w e b m d . c o m /$

${ }^{4} \mathrm{http}: / /$ www.nlm.nih.gov/medlineplus/
}

comprehension and empowerment and, consequently, improvement in the communication/interaction with medical professionals. With a specific focus on elderly people, the objective is to improve the overall communication between a user and the search engine so that he/she can easily express his/her requirements through everyday language and obtain the "right" and easy-to-understand amount of information.

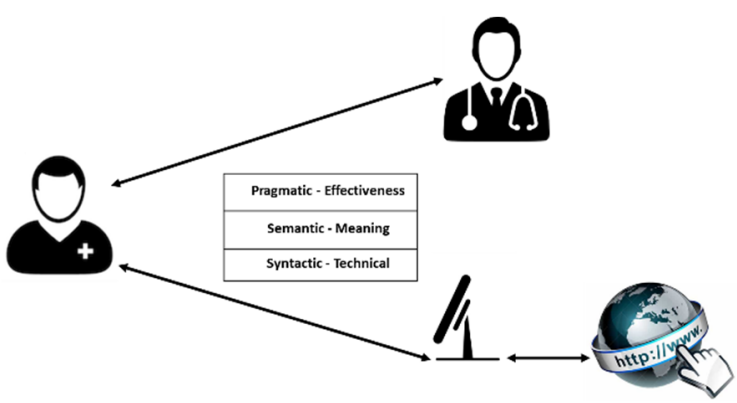

Figure 1: "Unified" communication model.

An analysis of the communication process of a generic search engine shows how it only works at the syntactic-technical level by retrieving Web page addresses (URLs) based on the keyword(s) specified by the user. It has not been designed to understand the user specific requirements (in the user own language) and, thus, it is only able to provide the user with generic information leaving him/her with the task of selecting, understanding and using the retrieved information (semantic and pragmatic part of the communication process). As a consequence, nonmedical experts, and especially elderly people, can be overwhelmed with the results and have great difficulties in the comprehension and use of the found information. This, in turn, reflects on their ability to have a "true" two-way communication with their doctors because, for example, they do not have a complete understanding of their medical conditions (semantic level)-when they do not misunderstand them-and then are unable to make shared and informed decisions (pragmatic level).

Since a generic search engine does not help much in the process of empowering patients/citizens, especially elderly ones, we have thought of creating a custom engine that allows a user to specify his/her information requirements in a simple way and provides the "right" amount of Web content, in a language that he/she can easily understand. This fully complies with all three levels of the communication model and provides a practical help to the empowerment process.

\footnotetext{
${ }^{5}$ http://www.hon.ch/
} 
The next sections present the characteristics and use of such a search engine, FACILE, that has been built in order to satisfy the user information needs and overcome the challenges confronted during the search process.

\section{A CUSTOM ENGINE FOR TAILORED RETRIEVAL OF HEALTH INFORMATION}

FACILE is a custom search engine specifically designed to facilitate the empowerment process of patients/citizens through the acquisition of knowledge online. Its objective is to support users in the health information seeking process on the Web according to their specific requirements.

The identification of the main requirements of the health information seekers on the Web has been carried out in (Alfano et al. 2019a) by analysing the works presented in (Pletneva et al. 2011; Banna et al., 2016; Roberts, 2017; Pian et al. 2017; Pang et al., 2015; Keselman, 2008). This literature review, although limited, has consistently shown the following main requirements of health information seekers:

- Language complexity;

- Information classification/customization;

- Information quality (mainly intended as information trustworthiness).

FACILE has been developed, based on these requirements by exploiting the semantic features of the Web and in particular those related to structured data and schema.org ${ }^{6}$ with particular reference to its health-lifesci extension ${ }^{7}$. The use of structured data on the Web is increasing over the years and the exploitation of structured data to collect information from the Web, in different sectors, has proven to be effective (Taibi et al. 2013, Dietze et al. 2017).

\subsection{Use of schema.org and health-lifesci Structured Data}

As said above, we have investigated how to leverage structured data to find suitable Web pages that satisfy the requirements of health information seekers. To this end, we have exploited the semantic information available in the World Wide Web and, in particular, the one provided by schema.org, an initiative funded

\footnotetext{
${ }^{6} \mathrm{https}: / /$ schema.org/

${ }^{7} \mathrm{https}: / /$ health-lifesci.schema.org/
}

by some major Web players, that aims to create, maintain, and promote schemas for structured data on the Internet. For the scope of the present work, we consider the health-lifesci extension that contains 93 types, 175 properties and 125 enumeration values related to the health/medical field.

We have performed an analysis of the healthlifesci elements using the data made available by the Web Data Commons initiative ${ }^{8}$. The Web Data Commons (WDC) (Meusel, 2014) contain all Microformat, Microdata and RDFa (Resource Description Framework in Attributes) data extracted from the open repository of Web crawl data named Common Crawl (CC) ${ }^{9}$. The data released in November 2018 have been used in this work. The whole dataset contains about 2.5 billion pages and about $37.1 \%$ of them contain structured data.

The dataset dump, used in our study, consists of 31.5 billion RDF n-quads ${ }^{10}$. These are sequences of RDF terms in the form $\{s, p, o, u\}$, where $\{s, p, o\}$ represents a statement consisting of subject, predicate, object, while $u$ represents the URI of the document from which the statement has been extracted. From the whole dataset, we have selected only the subset containing types, properties and enumeration values of health-lifesci.schema.org.

\subsection{Mapping Health Information Seeker Requirements to schema.org Elements}

When talking of online health information seekers, we can, mainly, consider two classes of users:

- Non experts (e.g., patients or citizens);

- Experts (e.g., physicians or medical researchers).

These two categories have different requirements, that can be connected to the language complexity and the other user requirements presented above. It is, then, important to understand which healthlifesci.schema.org elements can be mapped to the health information seeker requirements in order to use the structured data present in the Web to deliver tailored information to the user. In relation to the language complexity user requirement, healthlifesci.schema.org includes the MedicalAudience element that indicates whether the content is more suitable for a non-expert (Patient type) or an expert (Clinician and MedicalResearcher types, Alfano et

\footnotetext{
${ }^{8} \mathrm{http}: / /$ webdatacommons.org/

${ }^{9} \mathrm{http}: / /$ commoncrawl.org

${ }^{10} \mathrm{https}: / / \mathrm{www} . w 3.0 r g / \mathrm{TR} / \mathrm{n}$-quads/
} 


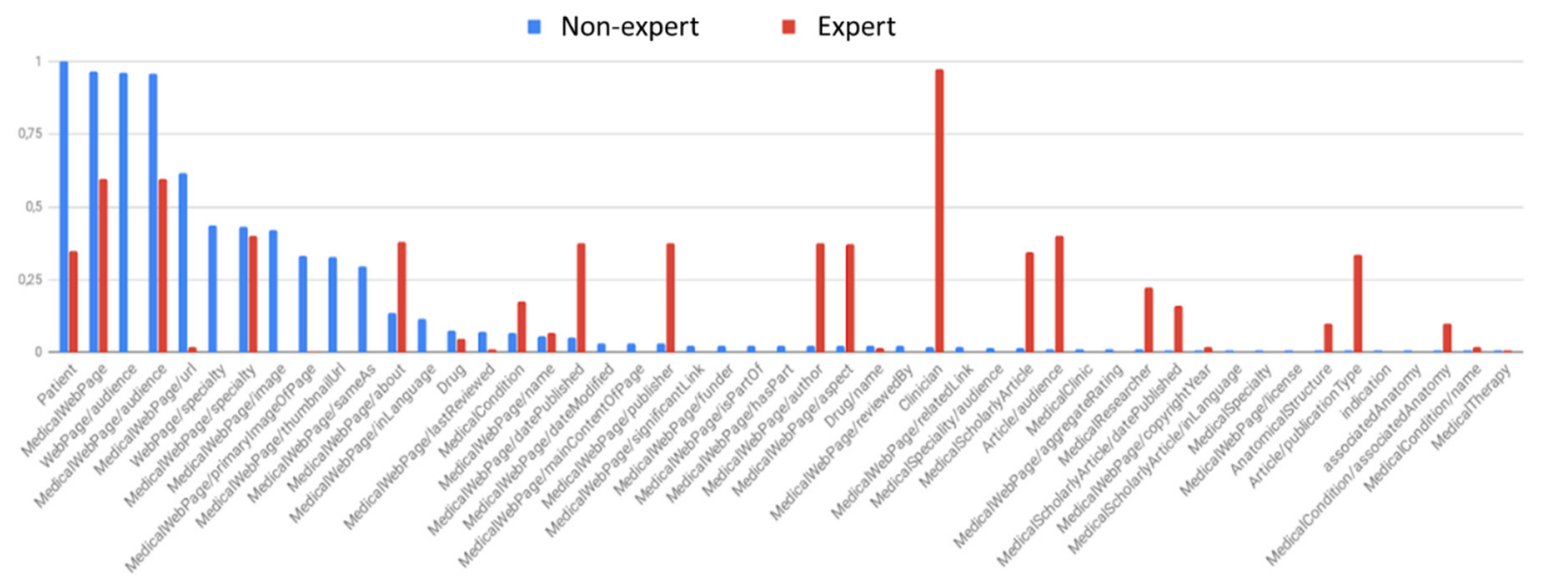

Figure 2: Comparison of most recurrent health-lifesci.schema.org elements for non-expert and expert audiences.

al., 2019c). A preliminary analysis on the dataset containing the health.life-sci.schema.org quadruples, shows that the distribution of schema.org elements heavily varies depending on the audience property of the pages (when specified). Figure 2 shows the comparison between the normalized distribution of the most recurrent health-lifesci.schema.org elements for non-expert and expert audiences.

In order to proceed with a classification and customization of information for the users, we have preliminarily analysed the most searched types of health/medical information on the Web, such as medical conditions, therapies and drugs (Taylor, 2010; Pew Research Center, 2013, Pletneva et al. 2011). We have then verified that these types appeared among the most recurring healthlifesci.schema.org elements reported in Fig. 2 in order to have enough data to be processed by FACILE and provide an effective information classification. At the end of this process, we have selected the following health.life-sci.schema.org elements for creating the classification of Web pages (the definitions are taken from https://healthlifesci.schema.org/):

- MedicalWebPage, indicates that the Web page provides medical information.

- MedicalScholarlyArticle, indicates that the Web page is a scholarly article in the medical domain.

- MedicalCondition, indicates any condition of the human body that affects the normal functioning of a person, whether physically or mentally.

- MedicalTherapy, indicates a medical intervention designed to prevent, treat, and cure human diseases and medical conditions.
- Drug, indicates a chemical or biologic substance, used as a medical therapy, that has a physiological effect on an organism. Used interchangeably with the term medicine.

- MedicalCode, provides a code for a medical entity.

- MedicalClinic, indicates a hospital or a medical school.

- MedicalSpecialty, indicates a specific branch of medical science or practice.

We have then taken the audience elements, i.e., Patient, Clinician and MedicalResearcher for the language complexity, the above health.lifesci.schema.org elements for the classification/customization of information and some schema.org elements related to data provenance (e.g., author and publisher) for the quality information (because the health-lifesci extension does not present such elements). This has brought us to create a mapping between the user requirements and the schema.org elements for the two user categories. This mapping expands the one presented in (Alfano et al., 2019a) and is reported in Table 1.

These schema.org elements are used by FACILE to retrieve Web pages and extract information based on the user specific requirements. As we will show in the next sections, by using FACILE, users can easily and quickly find the right amount of information that is reliable and, in a language suitable to their health literacy level, in full compliance with the three levels of the communication model presented above. This, in turn, improves their empowerment level and allows a better communication/interaction with the medical professionals. 
Table 1: Mapping between schema.org elements and user requirements for the "non-expert" and "expert" user categories.

\begin{tabular}{|c|c|c|c|}
\hline & Language Complexity & Information Classification & Information Quality \\
\hline Non-expert & $\begin{array}{l}\text { Audience: } \\
-\quad \text { Patient }\end{array}$ & \begin{tabular}{ll}
\multicolumn{2}{l}{ Type of document: } \\
$-\quad \quad$ MedicalWebPage \\
$-\quad$ WebPage \\
Topic Classification: \\
- $\quad$ MedicalCondition \\
$-\quad$ MedicalTherapy \\
$-\quad$ Drug \\
$-\quad$ MedicalCode \\
$-\quad$ & MedicalClinic \\
- & MedicalSpecialty
\end{tabular} & $\begin{array}{ll}\text { Reliability: } \\
-\quad & \text { author } \\
- & \text { publisher } \\
\text { - } & \text { lastRevised } \\
\text { - } & \text { datePublished }\end{array}$ \\
\hline Expert & $\begin{array}{l}\text { Audience: } \\
-\quad \text { Clinician } \\
-\quad \text { Medical Researcher }\end{array}$ & \begin{tabular}{ll}
\multicolumn{2}{l}{ Type of document: } \\
$-\quad$ & ScholarlyArticle \\
- & MedicalWebPage \\
& \\
Topic Classification: \\
$-\quad \quad$ MedicalCondition \\
$-\quad$ MedicalTherapy \\
$-\quad$ Drug \\
$-\quad$ MedicalCode \\
$-\quad$ MedicalClinic \\
$-\quad$ & MedicalSpecialty
\end{tabular} & \begin{tabular}{ll}
\multicolumn{2}{l}{ Reliability: } \\
$-\quad$ & author \\
- & publisher \\
- & lastRevised \\
- & datePublished
\end{tabular} \\
\hline
\end{tabular}

\subsection{Facile Implementation}

The mapping between user requirements and schema.org elements, shown in the previous section, has been used to build FACILE that provides the different audience types with the proper Web contents in terms of language complexity, information quality and information classification. It expands an initial version of the system that only takes into account the language complexity user requirement (Alfano et al., 2019c). Fig. 3 shows the user interface of the FACILE search engine available at the address http://www.math.unipa.it/simplehealth/faciles.

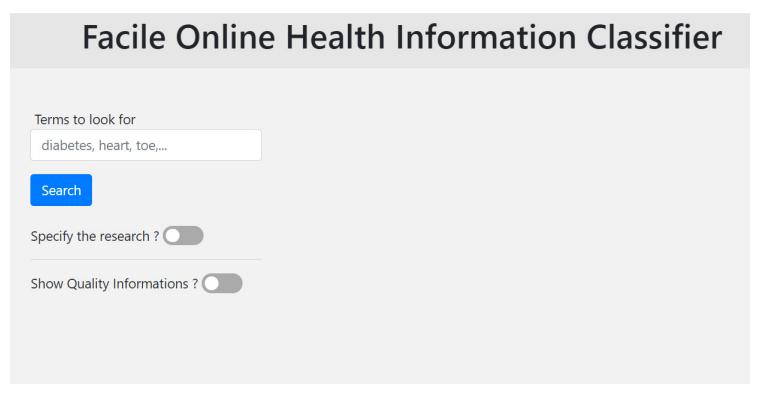

Figure 3: FACILE user interface.

The user interface includes a simple text input, similar to that of a generic search engine, where the user can insert the term(s) to be searched. Moreover, the user can decide to filter the results in order to get more focused information and not be overwhelmed with the amount of information that generic search engines provide. This is simply done through the two switch buttons shown in Fig. 3. The first switch button, "Specify the research", acts on the classification user requirement. It allows to search for either medical "Web Page" or for "Scholarly article" (Fig. 4). Medical Web pages usually present a mixed language and contain different types of information making them suitable to different target audiences. Scholarly articles are mostly research papers with a more technical language and mainly targeted to medical experts. The second switch button, "Show Quality Information", acts on the quality user requirement and provides information such as the last time the Web page has been reviewed, the publication date and the author or the publisher of the page (when available). This switch button does not filter out results, but it shows additional information useful to evaluate the information trustworthiness.

When selecting "Web Page", with the first switch button, some sub-filters appear in order to allow a classification of the Web pages in terms of the required type of medical information (Fig. 4). 


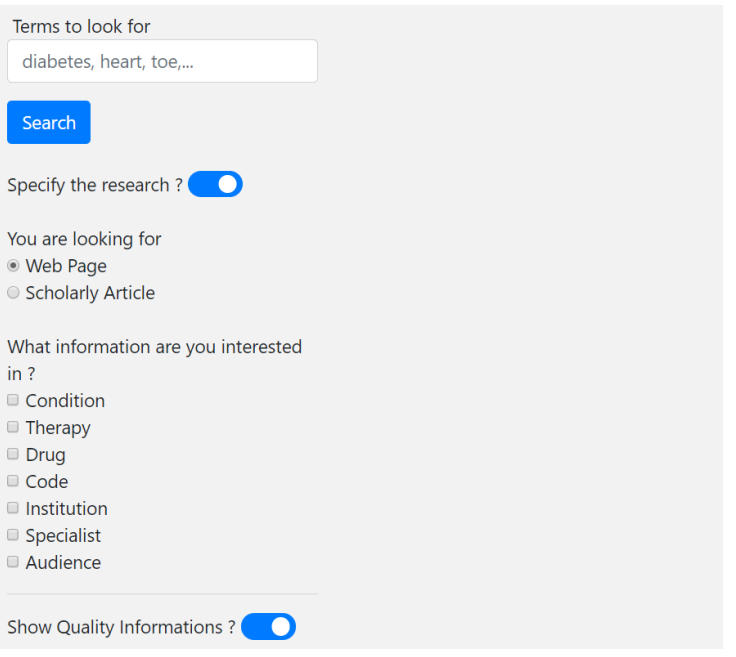

Figure 4: Web page sub-filters.

The Web Page sub-filters can be activated through checkboxes that can be easily selected by a user with no specific knowledge (such as an elderly patient), because they indicate common terms in the health domain. More than one checkbox can be selected each time and the filtering will be performed using the mapping of Table 1 and providing the following information:

- Condition: It will present the pages that contain a description of a medical condition and the values of the properties related to MedicalCondition schema.org element.

- Therapy: It will present the pages that contain information about a therapy and the values of the properties related to MedicalTherapy schema.org element

- Drug: It will present the pages that contain the information about a medicine and the values of the properties related to Drug schema.org element.

- Code: It will present the pages that contain the code of a medical condition and the values of the properties related to MedicalCode schema.org element. The code, together with the coding system, can be used to look on specialized website to find a specific condition/part of the body/therapy/drug, or other useful information.

- Institution: It will present the pages that contain the institutions (e.g., hospitals) that deal with a medical condition and the values of the properties related to MedicalClinic schema.org element.

- Specialist: It will present the pages that contain the values of the properties related to MedicalSpecialty schema.org element among which the medical specialists that deal with a medical condition.

- Audience: It will indicate the target audience of the Web page (patient/clinician/medical researcher) and the values of the properties related to MedicalAudience schema.org element. Finally, when a user is interested in finding more technical information, he/she can select "Scholarly Article". He/she will also have the possibility to specify a year in order to get the articles that have been reviewed that year or later (Fig. 5).

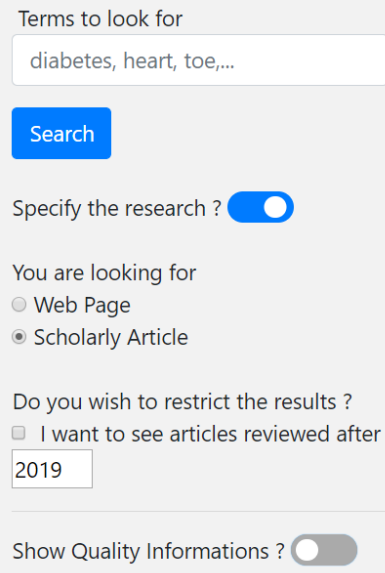

Figure 5: Scholarly Article sub-filters.

\section{EXPERIMENTAL USE}

FACILE, as shown in the previous section, has been designed and implemented to be used by different user typologies, i.e., medical experts or non-experts. Nevertheless, in relation to what discussed previously, we have executed some tests to evaluate FACILE effectiveness and usefulness for its use with non-experts and mainly with elderly people.

Preliminarily, notice that FACILE can be used as a generic search engine by inserting any keyword on the text input (Fig. 3) and receiving, at most, the top fifty results. Notice that a user usually analyses the first twenty-five-thirty results when using a generic search engine such as Google ${ }^{\mathrm{TM}}$ (Alfano et al. 2019b). Thus, from this point of view, the user is not penalized by using FACILE even though it is not using the whole Web but only the part that contains schema.org structured data. Thus, if we search for the hepatitis keyword, FACILE will provide the first fifty URLs that present the higher number of hepatitis occurrences (Fig. 6) 


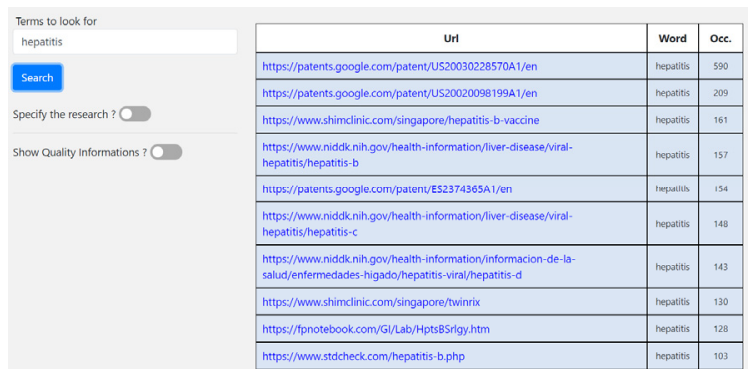

Figure 6: First results of generic search for hepatitis keyword.

The usefulness of FACILE can be appreciated as soon as the "Web page" filter and some of its sub-filters are used. In the example of Fig. 7, the "Condition" and "Code" sub-filters are checked. In this case FACILE provides just ten results so reducing the number of pages to be analysed and focusing to the Web content of interest. This allows an elderly patient, for example, to save a great deal of time by just focusing on the specific type of information he/she has requested (condition in this case).
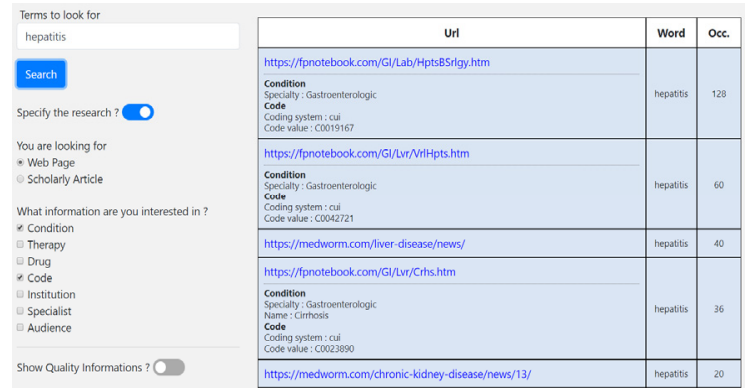

Figure 7: First results of search for hepatitis keyword with Condition and Code sub-filters.

Moreover, FACILE provides some information directly in the response page (e.g., Specialty: Gastroenterologic and Name: Cirrhosis for the Condition) that can be used for further investigation if of interest. Also the code identifier (e.g., CUI in the UMLS coding system) and code value (e.g., C0019167 that corresponds to Hepatitis $B e$ Antigens), can be used to have a unique reference of the information that is being read and that can be used for further discussion with a doctor or in a hospital. Notice that we plan to implement the translation of the code value in its corresponding term so to add this information directly in the response window.

If the user enables the "Show Quality Information?" switch button, the quality information, such as "Date published" or "Last reviewed", will be shown (Fig. 8) and the user will have the possibility to choose, for example, the most recent information.
If the author or publisher information are present, the user will have the possibility to analyse the information only if he/she trusts the source it is coming from.

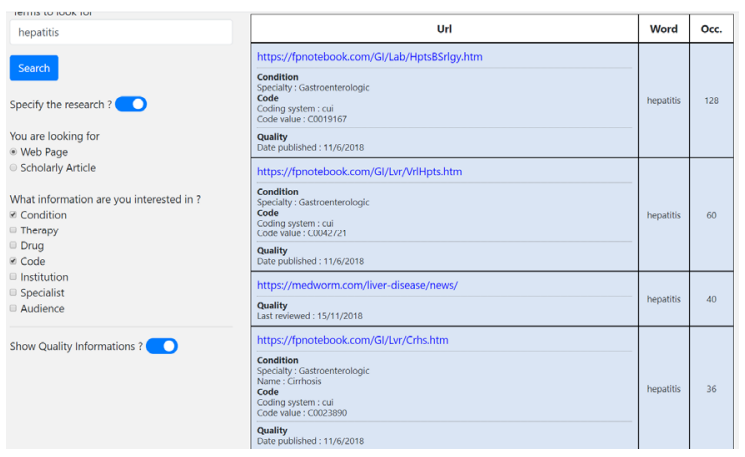

Figure 8: First results of search for hepatitis keyword with Quality filter activated.

If the user wants to find pages that match his/her medical literature level, he/she will check the "Audience" sub-filter. In this case the target audience of the page will be shown (whenever present - Fig. 9) and the user will be able to select the most suitable pages. For example, in the case of Fig. 9, an elderly patient would not analyse the pages whose audience is "clinician" so avoiding to waste time with Web content that is too difficult to understand.

Notice that in (Alfano et al., 2019c) we have evaluated the language familiarity of Web pages targeted to different audience types. This has been done by computing the "term familiarity index" of a word (i.e., number of results provided by the Google search engine, Kloehn, et al., 2018; Leroy, et al., 2012) and then computing the language familiarity of a Web page as the average of the term familiarity indexes of its words. The results clearly show that, on average, the Web pages targeted to patients have a much higher language familiarity, and thus a simpler terminology than the Web pages targeted to clinicians or medical researchers.

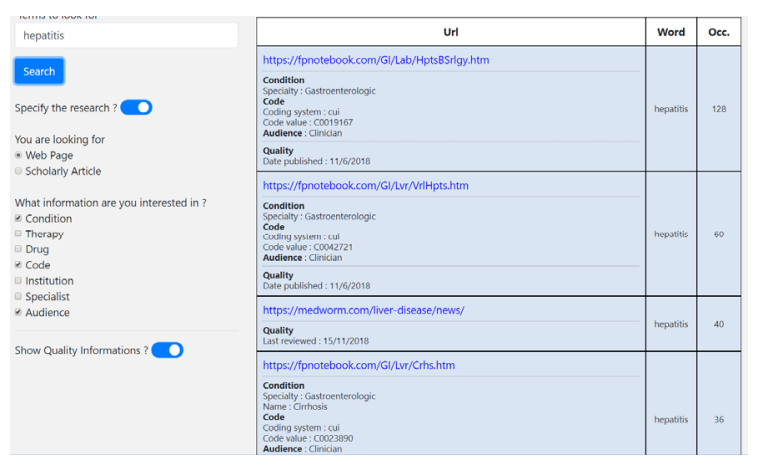

Figure 9: First results of search for hepatitis keyword with Audience sub-filter activated. 
For sake of completeness of all the filtering possibilities, we can finally assume that a medical expert is looking for information on hepatitis. In this case, given his/her skills, he/she will probably search for scholarly articles obtaining a result such as the one reported in Fig. 10 that provides more technical information.

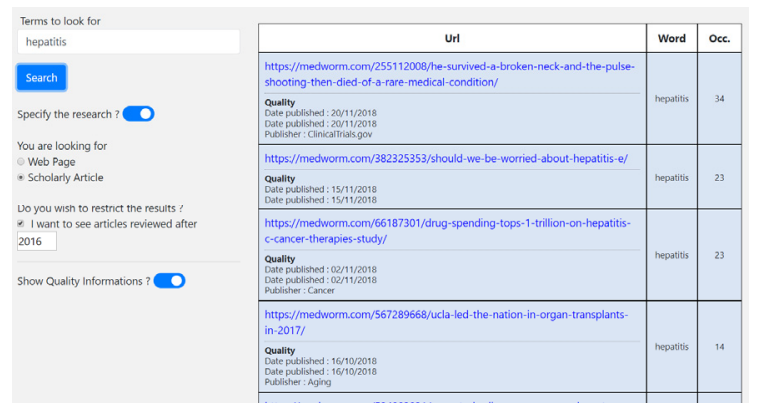

Figure 10: First results of search for hepatitis keyword with "Scholarly article" filter activated.

\section{DISCUSSION}

Although, the development of FACILE is still at prototypal stage and more experiments are undoubtedly needed, its principles and practical use show how FACILE complies with the three-level communication model presented in Section 2 allowing a non-expert user (e.g., an elderly patient) to specify his/her requirements in a simple language, translating into structured data elements for retrieving health information from the Web, and providing the user with the "right" amount of reliable and simple information that he/she can easily understand and employ in his/her process of empowerment and in subsequent communication/interaction with medical professionals. The compliance of Facile with the three levels of the communication model can be further analysed as follows:

1. Syntactic-Technical: FACILE presents the same retrieval capabilities as a generic search engine and, as such, it allows the user to search for health information on the Web (although it restrains the search to the semantic Web) and returns the requested information as any generic search engine does.

2. Semantic-Meaning: The translation capability of FACILE (mapping model) allows the user to specify his/her requirements in simple terms. FACILE translates the user requirements into schema.org elements in order to extract the appropriate information from the Web (communication phase: from user to FACILE).
Moreover, the ability of FACILE, to retrieve Web pages that present different language complexity levels, allows the user to choose the pages whose language can be easily understood (communication phase: from FACILE to user).

3. Pragmatic-Effectiveness: The response presented by FACILE has a pragmatic impact on the user (mainly a non-expert one) in terms of focused and reliable Web results (by avoiding to spend time analysing the large amount of results that a generic search engine provides), the specific information provided in the response page, and the language simplicity. The consequence is that a user is, overall, greatly facilitated in finding, understanding and using health/medical information on the Web and then in his/her empowerment process. This, among others, facilitates the comprehension of his/her medical conditions and increases his/her ability to communicate with medical professionals and make informed decisions.

Notice that the process of finding the "right" information may be iterative. Although, FACILE has the objective of immediately providing the user with the desired information, its flexibility and easiness of use allows the user to perform further searches when needed in order to get further information. For example, first he/she might need to understand a medical condition/disease and then find a therapy and the medicines for it. The user might also want to remove the filtering information, at some stage, in order to have the possibility of analysing more pages at the same time. Having already analysed the focused information (and related Web pages), he/she will have the ability and confidence to expand his/her exploration by quickly analysing the remaining information (or part of it). Overall, FACILE provides the user with the possibility of filtering and re-ranking the Web results according to his/her specific requirements but it leaves the user fully in charge of his/her navigational path on the Web. In this way, the user can freely and simply choose what he/she needs in terms of health/medical information, in any moment, so to achieve his/her empowerment objectives and act upon them. Notice that, although, most of the time, users search for health information on the Web before or after having consulted a medical professional as a doctor, the ideal case would see a doctor accompanying the patient in his/her navigational path on the Web so to help him/her to find the most suitable and reliable medical information. Of course, this is not always possible, due to time constraints. Nevertheless, we are working with medical professionals (doctors and nurses) to 
understand (and implement as much as possible) their advices to patients who navigate the Web in search of "good" health/medical information.

\section{CONCLUSIONS}

In this work, we have presented a "unified" threelevels communication model that allows a full interaction of a patient/citizen with both a machine (e.g., search engine) and another human (e.g., medical professional). We have shown as a generic search engine only reaches the first level of the communication model because it does not allow the user to specify his/her requirements and thus it does not provide focused information that the user can easily and promptly understand and use.

We have then presented FACILE, a custom search engine, that allows a user to specify his/her information requirements in a simple way and maps them to schema.org structured elements. It then retrieves the "right" and simple Web content without overwhelming the user. This positively affects his/her understanding and use and, as a consequence, the empowerment process.

The principles and first experimental results are satisfying and show FACILE potentialities even though, the used dataset (created with the 2018 structured data of Web Data Commons) has proven, sometimes, too limited in terms of provided results. Thus, we are in the process of adding the 2019 dataset (that has become available in the meantime) as well as the 2017 and 2016 datasets (the various datasets present some differences), so to have more data to experiment with and, hopefully, more results.

A deeper analysis also needs to be executed in order to better understand the mapping between the user requirements and the schema.org elements, as in the case of the information quality. Furthermore, although FACILE is simple and intuitive, we are in the process of running some tests with elderly people to evaluate their engagement level in using FACILE and understand what are the aspects that need to be improved. An evaluation of the reached empowerment level is also important and we are in the process of running some experiments with patients with multimorbidity. After using FACILE, the reached health literacy and empowerment levels will be measured.

As a future work, we want to further develop the unified communication model for improving the human-to-human and human-to-machine communication processes that underlie patient empowerment. For example, we plan to improve the communication between patients and medical professionals by translating the medical terms, retrieved by FACILE, in lay terms (Alfano et al., 2020; Alfano et al., 2018; Alfano et al., 2015). Moreover, we want to create a visual framework (Alfano et al., 2016) that uses the retrieving capabilities of FACILE and allows easy creation of advanced health services for elderly people, such as virtual assistants, thus facilitating the human-tomachine communication.

\section{ACKNOWLEDGEMENTS}

This work was partially supported by the European Union's Horizon 2020 research and innovation programme under the Marie Skłodowska-Curie grant agreement No 754489 and by Science Foundation Ireland grant 13/RC/2094 with a co-fund of the European Regional Development Fund through the Southern \& Eastern Regional Operational Programme to Lero, the Science Foundation Ireland Research Centre for Software, www.lero.ie.

We would like to thank Allan Gomes, of the Graduate School of Engineering of the University of Nantes, for his notable support in the technical development of the FACILE custom engine.

\section{REFERENCES}

Alfano, M., Lenzitti, B., Lo Bosco, G., Muriana, C., Piazza, T., Vizzini, G., 2020. Design, Development and Validation of a System for Automatic Help to Medical Text Understanding. International Journal of Medical Informatics, Elsevier. DOI:https://doi.org/10.1016/ j.ijmedinf.2020.104109

Alfano, M., Lenzitti, B., Taibi, D., Helfert, M., 2019a. Provision of Tailored Health Information for Patient Empowerment: An Initial Study. In Proceedings of the 20th International Conference on Computer Systems and Technologies (CompSysTech '19). Association for Computing Machinery, New York, NY, USA, 213220. DOI:https://doi.org/10.1145/3345252.3345301.

Alfano M., Lenzitti B., Taibi D., Helfert M., 2019b. ULearn: Personalized Medical Learning on the Web for Patient Empowerment. In: Herzog M., Kubincová Z. Han P., Temperini M. (eds) Advances in Web-Based Learning - ICWL 2019. ICWL 2019. Lecture Notes in Computer Science, vol 11841. Springer, Cham.

Alfano, M., Lenzitti, B., Taibi, D., Helfert M., 2019c. Facilitating access to health Web pages with different language complexity levels. Proc. of the 5th Inter. Conference on Information and Communication Technologies for Ageing Well and e-Health (ICT4AWE 2019), 2-4 May 2019, Heraklion-Crete. 
Alfano, M., Lenzitti, B., Lo Bosco, G., and Taibi, D., 2018. Development and Practical Use of a Medical Vocabulary-Thesaurus-Dictionary for Patient Empowerment. Proc. of ACM International Conference on Computer Systems and Technologies (CompSysTech'18), Ruse.

Alfano, M., Lenzitti, B., Lo Bosco, G., and Taibi, D., 2016. A Framework for Opening Data and Creating Advanced Services in the Health and Social Fields. Proc. of ACM International Conference on Computer Systems and Technologies (CompSysTech'16), Palermo.

Alfano, M., Lenzitti, B., Lo Bosco, G., and Perticone, V., 2015. An Automatic System for Helping Health Consumers to Understand Medical Texts, Proc. of HEALTHINF 2015, Lisbon, pp. 622-627.

Akerkar, S., \& Bichile, L., 2004. Health Information on the Internet: Patient Empowerment or Patient Deceit? Indian Journal of Medical Sciences, 58(8). Pp. 321326.

Banna, S., Hasan, H. \& Dawson, P., 2016. Understanding the diversity of user requirements for interactive online health services. International Journal of Healthcare Technology and Management, 15(3).

Bodolica, V., \& Spraggon, M., 2019. Toward patientcentered care and inclusive health-care governance: a review of patient empowerment in the UAE. Public Health, 169(971), 114-124.

Bravo, P., Edwards, A., Barr, P. J., Scholl, I., Elwyn, G., \& McAllister, M., 2015. Conceptualising patient empowerment: A mixed methods study. BMC Health Services Research, 15(1), 1-14.

Carlile, P., 2004. Transferring, Translating and Transforming: An Integrative Framework for Managing Knowledge Across Boundaries, Organization Science, 15(5), pp. 555-68.

Cerezo, P. G., Juvé-Udina, M. E., Delgado-Hito, P., 2016. Concepts and measures of patient empowerment: A comprehensive review. Revista Da Escola de Enfermagem.

Cherry, C. (1966). On human communication: a review, a survey, and a criticism. Cambridge, Mass, M.I.T. Press.

Dietze S., Taibi D., Yu R., Barker P., d'Aquin M., 2017. Analysing and Improving Embedded Markup of Learning Resources on the Web. Proc. of the 26th International Conference on World Wide Web Companion ( $W W W^{\prime} 17$ Companion), Switzerland, 283292.

European Health Parliament. 2017. PATIENT EMPOWERMENT AND CENTREDNESS.

Fumagalli, L. P., Radaelli, G., Lettieri, E., Bertele', P., Masella, C., 2015. Patient Empowerment and its neighbours: Clarifying the boundaries and their mutual relationships. Health Policy. 119(3), 384-394.

Hahn, L. K. \& Paynton, S. T. 2014. Survey of Communication Study. https://en.wikibooks.org/wiki/ Survey_of_Communication_Study.

Health promotion glossary. Geneva: World Health Organization; 1998.
Instituto Nacional de Estadística. 2010. Encuesta sobre Equipamiento y Uso de Tecnologías de la Información y Comunicación en los hogares.

Johnson, F.C., Klare, G.R. 1961. General Models of Communication Research: A Survey of the Developments of a Decade, Journal of Communication, Volume 11, Issue 1, March 1961, Pages 13-26.

Keselman, A., Logan, R., Smith, C. A., Leroy, G., \& ZengTreitler, Q., 2008. Developing informatics tools and strategies for consumer-centered health communication. Journal of the American Medical Informatics Association : JAMIA, 15(4), 473-483.

Kloehn, N. et al., 2018. Improving consumer understanding of medical text: Development and validation of a new subsimplify algorithm to automatically generate term explanations in English and Spanish. Journal of Medical Internet Research, 20(8).

Leroy, G. et al., 2012. Improving perceived and actual text difficulty for health information consumers using semiautomated methods. AMIA Annual Symposium Proceedings. pp.522-31.

Morris, C.W. 1938. Foundations of the theory of signs. International encyclopedia of unified science, vol. 1, no. 2. The University of Chicago Press, Chicago.

Meusel, R., Petrovski, P., and Bizer, C. 2014. The WebDataCommons Microdata, RDFa and Microformat Dataset Series. Proc. of the 13th International Semantic Web Conference (ISWC14), Springer-Verlag New York, USA, 277-292.

Pang, P. C.-I.; Verspoor, K.; Pearce, J.; Chang, S. 2015. Better Health Explorer: De-signing for Health Information Seekers. In OzCHI '15 Proceedings of the Annual Meeting of the Australian Special Interest Group for Computer Human Interaction (pp. 588-597).

Pletneva, N., Vargas, A. \& Boyer, C., 2011. Requirements for the general public health search. Khresmoi Public Deliverable D8.1.1.

Pew Research Center, 2013. Health online 2013 , http://www.pewinternet.org/2013/01/15/health-online2013/.

Pian, W., C.S.G. Khoo, J. Chi. 2017. Automatic classification of users' health information need context: Logistic regression analysis of mouse-click and eyetracker data. Journal of Medical Internet Research, 19(12).

Roberts, T. 2017. Searching the Internet for Health Information: Techniques for Patients to Effectively Search Both Public and Professional Websites. SLE Workshop at Hospital for Special Surgery Tips For Evaluating the Quality of Health, 1-12.

Shannon, C., W. Weaver. 1949. The Mathematical Theory of Communications. University of Illinois Press, Urbana, IL

Smith, T. 2004. Exploring the characteristics of active health seekers, the thinking behind patient preferences, and the implications for patient-professional relationships. Quality and Safety in Health Care, 13(6), 474-477.

Taibi D., Fetahu B., Dietze S. 2013. Towards integration of web data into a coherent educational data graph. In 
Proc. of the 22nd Int. Conference on World Wide Web (WWW'13 Companion). Association for Computing Machinery, New York, NY, USA, 419-424.

Taylor, H. 2010. HI-Harris-Poll-Cyberchondrics. Harris Interactive. https://theharrispoll.com/the-latest-harrispoll-measuring-how-many-people-use-the-internet-tolook-for-information-about-health-topics-finds-thatthe-numbers-continue-to-increase-the-harris-poll-firstused-the-word-cyberch/.

UK national statistics, 2010. Statistical bulletin: Internet Access 2010. Office for National Statistics. 27 Aug 2010.

Watzlawick, P., Beavin, J. B., \& Jackson, D. D. 1967. Pragmatics of human communication: a study of interactional patterns, pathologies, and paradoxes. Norton, New York.

World Health Organization (WHO). 2016. Framework on integrated, people-centred health services: Report by the Secretariat. World Health Assembly, (A69/39), 112.

World Health Organization (WHO). 1998. Health promotion glossary. https://www.who.int/ healthpromotion/about/HPR\%20Glossary\%201998.pd f. 